PSFC/JA-01-23

\title{
2D Images of Deuterium Emission in the Alcator C-Mod Tokamak Divertor
}

\author{
C.J. Boswell, J.L. Terry
}

October 2001

Plasma Science and Fusion Center Massachusetts Institute of Technology

Cambridge, MA 02139 USA

This work was supported by the U.S. Department of Energy, Cooperative Grant No. DE-FC02-99ER54512. Reproduction, translation, publication, use and disposal, in whole or in part, by or for the United States government is permitted.

Submitted for publication to IEEE Transactions in Plasma Science. 


\title{
2D Images of Deuterium Emission in the Alcator C-Mod Tokamak Divertor
}

\author{
Christopher J. Boswell, James L. Terry
}

\begin{abstract}
Two $7 \mathrm{~mm}$ diameter, remote-head, visible, charge-coupled device (CCD) cameras are used on the Alcator C-Mod tokamak to generate two-dimensional emissivity profiles of deuterium line radiation (typically at Balmer $\alpha$ and Balmer $\gamma$ ) from the divertor. These emissivity profiles are obtained by reconstructing the measured brightnesses using the thin-chord approximation and assuming toroidal symmetry of the emission. The regions of the divertor with a high ratio of Balmer $\gamma$ to Balmer $\alpha$ emission are indicative of a plasma that is recombining. These recombining regions in the divertor are seen to shift from the inner to the outer divertor as the core density in increased.
\end{abstract}

\section{Keywords}

Alcator C-Mod, divertor, deuterium emission, tomographic reconstruction

LCATOR C-Mod is a high magnetic field, high density, compact tokamak, with a parallel heat flux to 1 the divertor as high as $600 \mathrm{MWm}^{-2}$ [1]. This tokamak has operated with a toroidal magnetic field at the magnetic axis of up to 8 Tesla (5.4 Tesla typically), a core line-averaged electron density of up to $3 \times 10^{21} \mathrm{~m}^{-3}$ ( $2 \times 10^{20} \mathrm{~m}^{-3}$ typically), and a plasma current of $1.5 \mathrm{MA}$ (1 MA typically). Its major and minor radii are $0.67 \mathrm{~m}$ and $0.22 \mathrm{~m}$ respectively. High toroidal magnetic field and high density in the core of the tokamak allow Alcator C-Mod to explore regions of dimensionless parameters not typically accessible with other tokamaks. The high parallel heat flux allows a direct simulation of the conditions expected in a magnetically confined fusion reactor. The places where this high heat flux typically strike a material surface in Alcator C-Mod are the divertor plates, which are magnetically isolated from the core plasma.

The purpose of a divertor is to alleviate the problem of impurity contamination in the core region of the plasma. The divertor accomplishes this by moving the hot plasma-surface interactions away from the plasma core and by providing a region of higher pressure neutrals where impurities and He "ash" can be pumped out of the vacuum vessel. Figure 1 shows a cross-section of the divertor region of Alcator C-Mod. The red curves represent the last closed flux surface, or separatrix, which is the magnetic boundary of the hot core plasma. The divertor region is the general name given to the entire area shown in the figure. The plasma that escapes the core typically flows along the magnetic field toward the structure shown in blue. The structures where the plasma strikes are known as the divertor plates and are made of a material with a high melting temperature (in this case Molybdenum) so that they can withstand the high heat fluxes.

Although the Alcator C-Mod divertor can handle the high heat fluxes in pulsed experiments a magnetically confined fusion power plant will need to handle these heat fluxes in steady state. Currently no uncooled materials can handle the expected heat flux in steady state and therefore, a method of dissipating this power before it arrives at the divertor plates is essential. One method of dissipating the heat flux is to create a so-called detached plasma in the divertor region. In detached divertor operation the heat flux to the divertor plates is reduced through line radiation, ion-neutral friction, and/or volumetric plasma recombination that occur "upstream" of the plates. The detached divertor is characterized by a low temperature $\left(T_{e} \approx 2 \mathrm{eV}\right)$, and high density $\left(n_{e} \approx 10^{21} \mathrm{~m}^{-3}\right)$ plasma existing above the divertor plates, where most of the heat flux and particle current is reduced before striking the divertor plates. Therefore, it is important to know the plasma parameters in this region to understand better and control the detached divertor condition. By measuring deuterium emission when the divertor is detached it is possible to obtain electron temperature (using the relative intensities of the Balmer series originating from the high- $n$ levels which are dependent on electron temperature [2]), and electron density (using the Stark-broadening of the Balmer series lines). By knowing these quantities and a spatial distribution of the $n=5 \rightarrow 2\left(\mathrm{D}_{\gamma}\right)$ deuterium emission, a volumetric recombination rate in the divertor can be determined.

On Alcator C-Mod there are two tangentially viewing CCD cameras used in the analysis of emission distribution in the divertor region [3]. Both CCD cameras are off-the-shelf remote-head "pencil" cameras. The 
cameras are $7 \mathrm{~mm}$ in diameter and $40 \mathrm{~mm}$ in length. A $3 \mathrm{~m}$ cable connects the cameras to the electronics controlling their output. Both cameras are mounted in aluminum holders and affixed to a G-10 platform inside a reentrant tube and behind a shuttered quartz window $10 \mathrm{~cm}$ from the last closed flux surface of the Alcator C-Mod plasma. Being mounted in the reentrant tube places the cameras inside the toroidal field coils and therefore exposed to magnetic fields of up to 6 Tesla. An interference filter with a bandwidth of $9 \mathrm{~nm}(4 \mathrm{~nm})$ and with a central wavelength of $661 \mathrm{~nm}(437 \mathrm{~nm})$ and is placed just in front of each camera. The cameras and filters are absolutely calibrated and the images are tomographically reconstructed using a thin-chord approximation and assuming toroidally symmetric emission to obtain two-dimensional emissivity profiles. The tangential view gives both a vertical and a horizontal information to the reconstructed images.

Figure 1 shows the tomographic reconstructions of $\mathrm{D}_{\gamma}$ emissivity obtained from the CCD camera measurements. The four reconstructions are from four different line-averaged core electron densities. Figure 1(a) is a low density case where all of the $\mathrm{D}_{\gamma}$ emission comes from the inner divertor plate region. Figure 1(b) is a moderate density case where most of the $\mathrm{D}_{\gamma}$ emission is in the private flux zone, the region below where the last closed flux surface is in the shape of an "x." Figure 1(c) is at the density where the outer divertor plate begins to detach. In this case the $\mathrm{D}_{\gamma}$ emission is concentrated at the outer strike-point (where the last closed flux surface intersects the outer divertor plate). Figure 1(d) is at a higher density when the detached region above the outer divertor plate is much larger. Here the $\mathrm{D}_{\gamma}$ emission is spread from the outer strike-point to the x-point. Through spectroscopic measurements this region in known to be a cold $\left(T_{e}<1 \mathrm{eV}\right)$, dense $\left(n_{e} \approx 10^{21} \mathrm{~m}^{-3}\right)$ plasma.

The cause for the shifting of the $\mathrm{D}_{\gamma}$ emission from being at the inner divertor plate to being predominantly at the outer divertor plate is attributed to a cross-field $\mathbf{E} \times \mathbf{B}$ drift generated by an electron temperature gradient along the flux surface of the outer scrape-off layer, for more details see [4]. In the low density case, see figure 1(a), the temperature gradient along a field line is shallow due to the fact that there is no significant heat loss processes occurring. This shallow gradient does not generate a strong enough electric field for the perpendicular $\mathbf{E} \times \mathbf{B}$ drift to drive significant particle flux into the private flux zone. As the electron density is further increased, see figure 1(b), the neutral deuterium recycling from the material surfaces generates enough neutrals that a strong temperature gradient can be formed between the x-point and the outer strike-point. This strong temperature gradient does produce a significant flux of plasma into the private flux zone where it recombines and generates $\mathrm{D}_{\gamma}$ emission. As the density is increased further, see figure 1(c) a detachment front is formed above the outer divertor plates. Behind the front the plasma is cold and dense and is strongly recombining. Ahead of the front a temperature gradient still exists and still drives plasma into the private flux zone from the outer leg. Once the density is increased even further, see figure 1(d), the detachment front moves all the way to the x-point leaving behind it a region of cold, dense, strongly recombining, and shallow temperature gradient plasma yielding a large region of $\mathrm{D}_{\gamma}$ emission.

\section{ACKNOWLEDGMENTS}

This work was supported by the US Dept. of Energy under grant \#DE-FC02-99ER54512

\section{REFERENCES}

[1] I. H. Hutchinson, R. Boivin, F. Bombarda, P. Bonoli, S. Fairfax, C. Fiore, J. Goetz, S. Golovato, R. Granetz, M. Greenwald, S. Horne, A. Hubbard, J. Irby, B. LaBombard, B. Lipschultz, E. Marmar, G. McCracken, M. Porkolab, J. Rice, J. Snipes, Y. Takase, J. Terry, S. Wolfe, C. Christensen, D. Garnier, M. Graf, T. Hsu, T. Luke, M. May, A. Niemczewski, G. Tinios, J. Schachter, and J. Urbahn, "First results from alcator c-mod," Physics of Plasmas, vol. 1, no. 5, pp. 1511-1518, May 1994.

[2] Hans R. Griem, Principles of Plasma Spectroscopy, vol. 2 of Cambridge Monographs on Plasma Physics, Cambridge University Press, Cambridge, UK, 1997.

[3] C. J. Boswell, J. L. Terry, B. Lipschultz, and J. Stillerman, "Applications of visible ccd cameras on the alcator c-mod tokamak.," Review of Scientific Instruments, vol. 72, no. 1, pp. 935-939, January 2001.

[4] C. J. Boswell, J. L. Terry, B. LaBombard, B. Lipschultz, and J. A. Goetz, "Observations of cold, high density plasma in the private flux region of the alcator c-mod divertor," Journal of Nuclear Materials, vol. 290-293, pp. 556-560, 2001. 


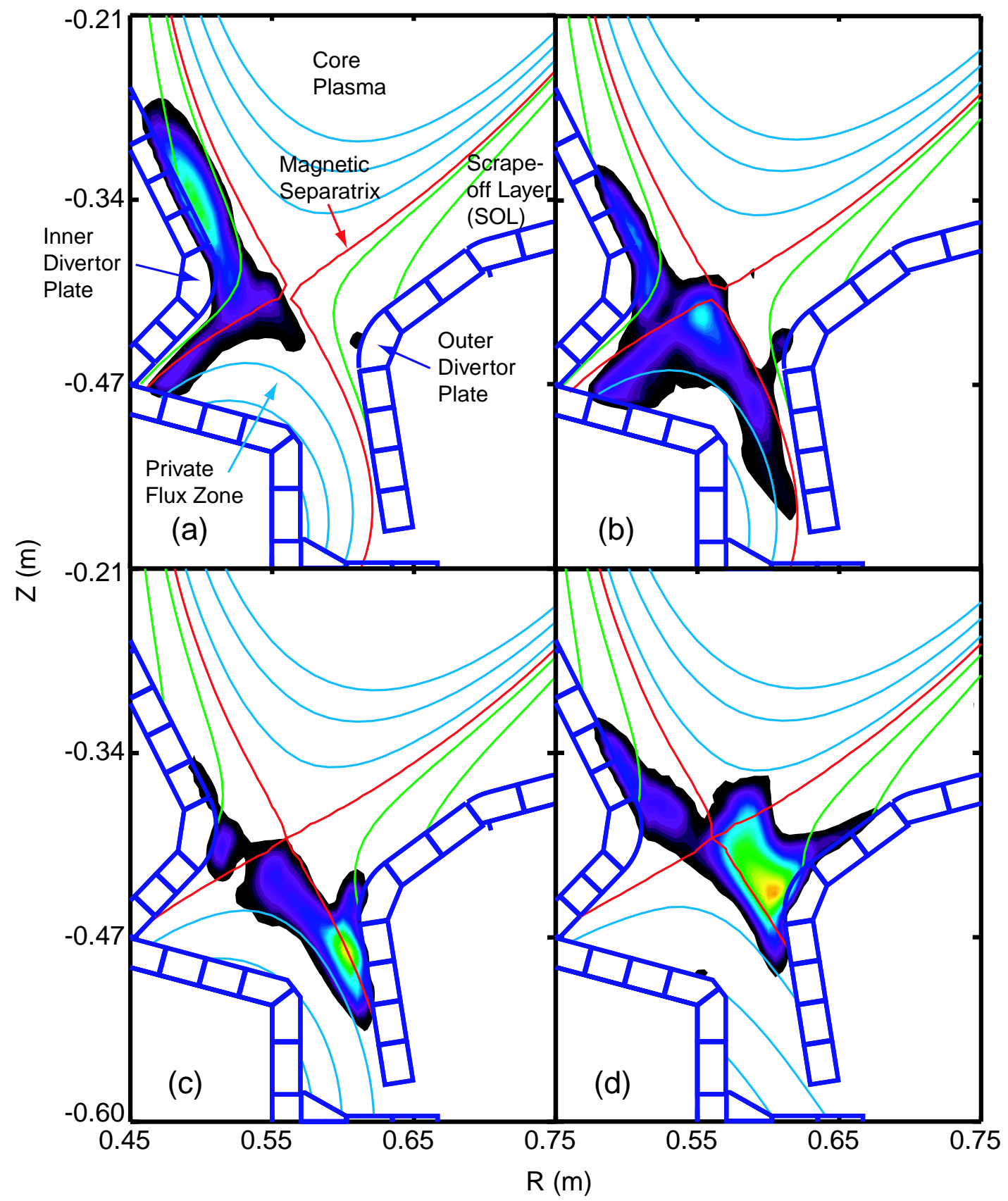

15.

11.

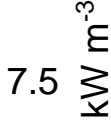

3.7

0.0

Fig. 1. Four tomographically reconstructed emission profiles of deuterium Balmer $\gamma$ increasing tokamak density, (a) line averaged electron density of $1.16 \times 10^{20} \mathrm{~m}^{-3}$, (b) line averaged electron density of $1.66 \times 10^{20} \mathrm{~m}^{-2}$, (c) line averaged electron density of $1.86 \times 10^{20} \mathrm{~m}^{-3}$, and (d) line averaged electron density of $2.30 \times 10^{20} \mathrm{~m}^{-3}$. 